\title{
OPTIMIZATION OF RF COMPRESSOR IN THE SPARX INJECTOR
}

\author{
M. Boscolo, M. Ferrario, B. Spataro, INFN-LNF Frascati (Rome); C. Ronsivalle, ENEA-Frascati \\ (Rome); L. Serafini,INFN-MI, Milan
}

\section{Abstract}

The SPARX photoinjector consists in a rf gun injecting into three SLAC accelerating sections, the first one operating in the RF compressor configuration in order to achieve higher peak current. A systematic study based on PARMELA simulations has been done in order to optimize the parameters that influence the compression also in view of the application of this system as injector of the so called SPARXINO 3-5 nm FEL test facility. The results of computations show that peak currents at the injector exit up to $\mathrm{kA}$ level are achievable with a good control of the transverse and longitudinal emittance by means of a short SW section operating at $11424 \mathrm{MHz}$ placed before the first accelerating section. Some working points in different compression regimes suitable for FEL experiments have been selected. The stability of these points and the sensitivity to various types of random errors are discussed.

\section{SPARX INJECTOR}

The first phase of the SPARX project [1] consists in developing techniques and critical components for future $\mathrm{X}$-ray facilities. One of the lines of this R\&D activity foresees the use of the SPARC photoinjector [2] to test $\mathrm{RF}$ compression techniques aimed to the generation of electron beams with high peak brightness.

The SPARC photoinjector, now under construction in Frascati, consists of a 1.6 cell RF gun operated at S-band with a peak field on the cathode of $120 \mathrm{MV} / \mathrm{m}$ and an incorporated metallic photo-cathode followed by an emittance compensating solenoid and three accelerating sections of the SLAC type (2856 MHz travelling wave), the first one embedded in a solenoid composed by an array of 13 coils. It is aimed to provide a $155 \mathrm{MeV}-100 \mathrm{~A}$ bunch with a projected emittance less than $2 \mu \mathrm{m}$ and a slice emittance less than $1 \mu \mathrm{m}$ for the $50 \%$ of slices driving a saturating SASE $500 \mathrm{~nm}$ FEL without the use of a compressor scheme.

For the SPARX RF compression tests it is planned to use the first SPARC accelerating section as RF compressor, to add a solenoid on the sections \#2 and \#3 and to place a $11424 \mathrm{MHz}$ (the 4th harmonic of the operating frequency of the TW sections) short linac before the first accelerating structure.

\section{RF COMPRESSOR OPTIMIZATION}

RF compressor techniques, based on the simultaneous action of velocity bunching and emittance compensation have been theoretically and numerically studied [3] and partially confirmed by experimental results [4]. The SPARX injector will be devoted to perform optimized velocity bunching studies and to provide definitive tests of the usefulness of this technique. A systematic study based on extensive numerical simulations of beam dynamics in the SPARX injector has been done by PARMELA code [5] in order to optimize all the parameters and to study the stability of the system.

\section{No IV Harmonic Section Included}

A first set of PARMELA runs was done to determine the dependence of the final current from the phase on RF compressor and to optimize the magnetic fields in the solenoids embedding the accelerating sections in order to minimize the emittance in different compression regimes without the use of the X-band section. The results, summarized in table 1 , demonstrate that it is possible to reach good levels of compression with a good control of emittance, but some undesired characteristics limit the practical use of RF compression especially in view of a further compression at higher energy.

Table 1: RF compressor parameters

\begin{tabular}{|c|c|c|c|}
\hline $\begin{array}{c}\mathbf{R F} \\
\text { compressor } \\
\text { phase range }\end{array}$ & $\begin{array}{c}\text { B1,B2,B3 } \\
\text { (gauss) }\end{array}$ & $\begin{array}{c}\text { Current } \\
\text { (A) }\end{array}$ & $\begin{array}{c}\text { Max. } \\
\text { Emittance } \\
(\mu \mathbf{m})\end{array}$ \\
\hline$-60^{\circ} /-75^{\circ}$ & $1200,0,0$ & $117-151$ & 0.7 \\
\hline$-75^{\circ} /-83^{\circ}$ & $1200,1400,0$ & $151-249$ & 0.8 \\
\hline$-83^{\circ} /-87^{\circ}$ & $1200,1400,0$ & $249-458$ & 1.3 \\
\hline$-87^{\circ} /-91^{\circ}$ & $\begin{array}{c}\text { Ramped from } \\
1200 \text { to } 1800\end{array}$ & $458-1180$ & 2.8 \\
\hline
\end{tabular}

In fact the increase of compression gives: 1) an increasing deformation in the shape of the bunch that appears as a spike followed by a long tail 2) a highly non linear longitudinal phase space 3) a strong sensitivity to phase jitter that gives for compression factors greater than 3 a percentage variation of current of $\sim 15-25 \%$ for an error phase of $1 \mathrm{deg}$ on the RF compressor. These effects are due to the combination of the RF and space charge non-linearities. A partial compensation can be obtained by the use of the foreseen IV harmonic section placed between the gun and the RF compressor.

\section{Harmonic Section Included}

With the $11424 \mathrm{MHz}$ section switched on, the optimization criterion is to bunch the beam in the centre with a linear correlation phase-energy differently from the unlinearized case where the charge piles up in the bunch head and only a portion of the electrons (typically the half) is involved by the compression process.

From simple analytical considerations the beam energy should be decreased of $1 / 16$ of the energy gain in the RF 
compressor, but due to deceleration the input energy and energy spread change give different energy gain and phase slippage in the SLAC sections, so that the amount of the needed deceleration must be determined numerically. From PARMELA simulations it results that the beam energy must be reduced from $5.64 \mathrm{MeV}$ to $\sim 3.3$ $\mathrm{MeV}$, that can be obtained by different combinations of phase and amplitude (Ex) in the $\mathrm{X}$-band section, giving different compression factors for the same phase on the $\mathrm{RF}$ compressor. As it is shown in figure 3 the beam current can be raised to $\sim 950 \mathrm{~A}$.

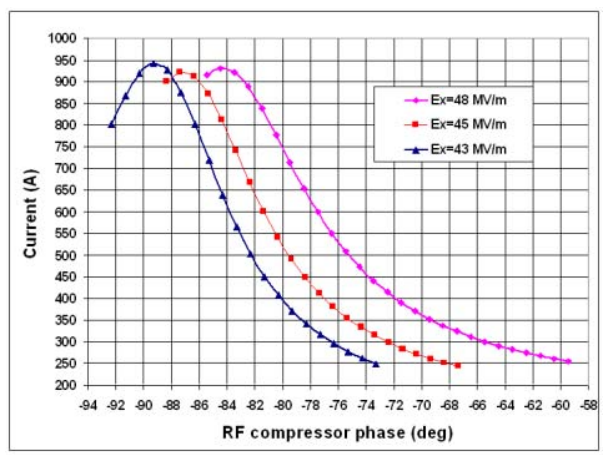

Figure 1: Final current vs RF compressor phase for different electric fields amplitude in the $\mathrm{X}$. band section and the same decelerating voltage.

In the range 300-900 A these curves have been fitted by a $4^{\text {th }}$ order polynomial whose derivative gives the sensitivity to the RF compressor phase jitter: in figure 4 the quantity $(\mathrm{dI} / \mathrm{d} \varphi) / \mathrm{I}$ is plotted vs the beam current for these three curves compared with the case of compression without IV harmonic section. The sensitivity to phase jitter is reduced of a factor $\geq 2$ by the use of the IV harmonic section.

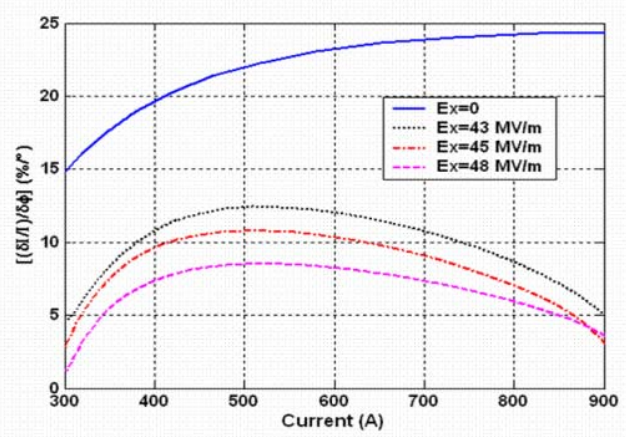

Figure 2: The percentage variation of current for an error of 1 degree in the RF compressor phase.

\section{WORKING POINTS IN MEDIUM AND HIGH COMPRESSION REGIME}

In the parameters space of figure 3 two working points have been selected also in view of the application of the system as injector of the so-called SPARXINO 3-5 nm FEL test facility [1] based on an upgrade of the actual Frascati $800 \mathrm{MeV}$ linac including a second stage of compression of magnetic type.
The first working point (fig.3) is particularly suitable to a further magnetic compression due to its good beam characteristics in terms of current $(450 \mathrm{~A})$ and emittance $(1.03 \mu \mathrm{m})$ and good linearity of $\varphi$-E space compared with the case in which the same average current is obtained with the IV harmonic section off (fig.4).

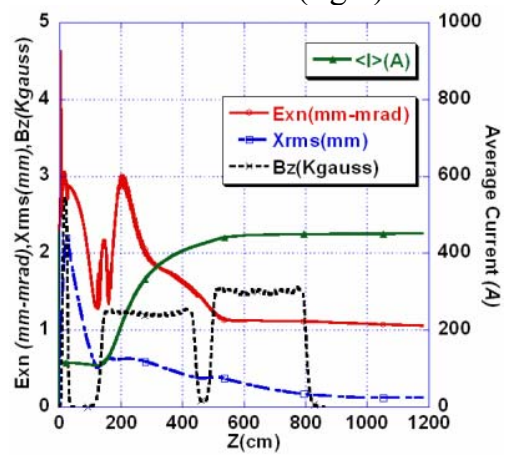

Figure 3: Average current, transverse emittance and envelope, axial magnetic field vs $\mathrm{z}$ for a 450 A compressed beam.

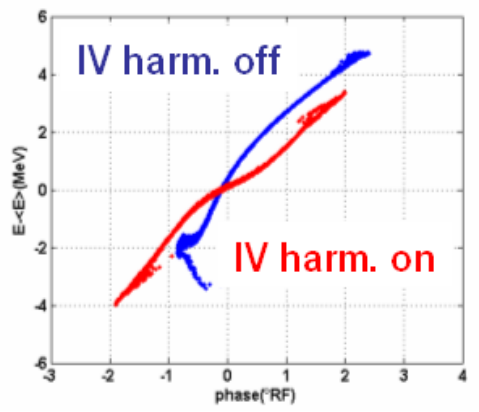

Figure 4: Longitudinal phase space for a 450 A compressed beam with and without the IV harmonic section.

The relative slice analysis for a slice length of $30 \mu \mathrm{m}$ shown in figure 5 indicates that the compression occours in the central part of the bunch, where the energy spread and emittance are at a minimum, that maximizes the normalized brightness, $\mathrm{B}_{\mathrm{n}}=\mathrm{I} /\left(4 \pi \varepsilon_{\mathrm{nx}} \varepsilon_{\mathrm{ny}}\right)$, that reaches $6 \cdot 10^{13}$ for the central slice.
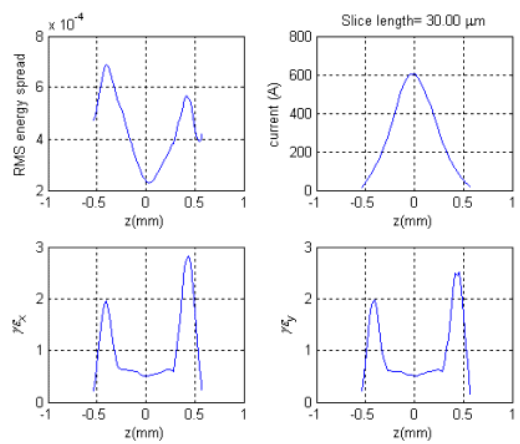

Figure 5: Slice analysis for a $450 \mathrm{~A}$ compressed beam.

The second working point (figs. 6,7) is interesting for its high current $(860 \mathrm{~A})$ and very good control of the projected $(1.5 \mu \mathrm{m})$ and slice emittance allowing to rise the peak slice brightness to $1.13 \cdot 10^{14}$. 


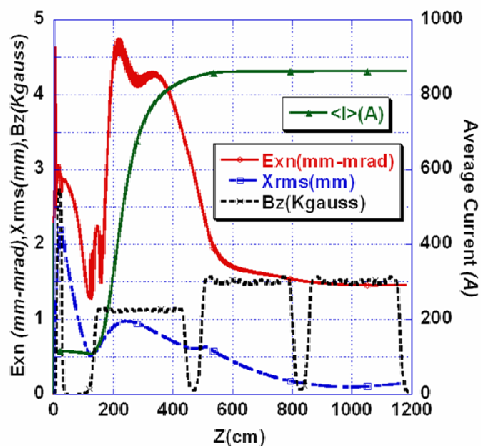

Figure 6: Average current, transverse emittance and envelope, axial magnetic field vs $\mathrm{z}$ for a 860 A compressed beam.
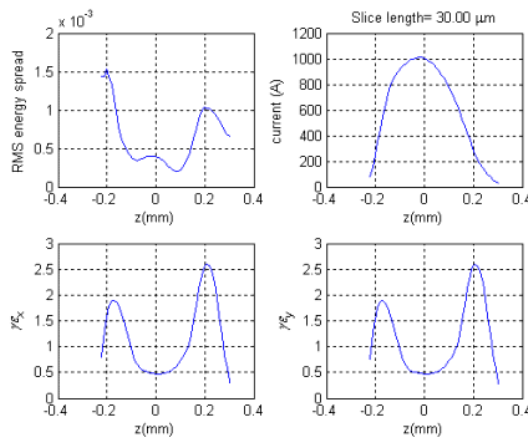

Figure 7: Slice analysis for a 860 A compressed beam.

\section{SENSITIVITY TO ERRORS}

The sensitivity of the two working points described above to various types of random errors under realistic conditions has also been studied. Following a technique already used for the SPARC working point described in [6], a statistical analysis has been done based on the results of one hundred PARMELA runs performed for each working point, each one with random errors set within the limits of table 2 using the criterion of having a maximum growth of the projected emittance of $10 \%$ and a maximum current variation of $\pm 15 \%$ with respect to the nominal case.

Table 2: Variation of parameters for combined random errors study in the SPARX injector

\begin{tabular}{|c|c|}
\hline Parameter & Error range \\
\hline Phase jitter gun-linac & $\pm 1^{\circ}$ \\
\hline Phase jitter gun-X band section & $\pm 1^{\circ}$ \\
\hline Charge fluctuation & $10 \%$ \\
\hline Gun B field amplitude & $\pm 0.4 \%$ \\
\hline Gun E field amplitude & $\pm 0.5 \%$ \\
\hline Spot radius & $\pm 10 \%$ \\
\hline Spot ellipticity & $3.5 \%$ \\
\hline
\end{tabular}

The results of the simulations were used to construct the curves plotted in fig. 8 that give the probability to obtain an emittance greater or equal than the corresponding value on the abscissa for the two considered compressed beams compared with the noncompressed SPARC beam. For example a probability of $10 \%$ corresponds to a normalized projected emittance $\geq 1$ $\mu \mathrm{m}$ at $100 \mathrm{~A}$ and to $\sim 1.8 \mu \mathrm{m}$ at $450 \mathrm{~A}$ and to $\sim 2.25 \mu \mathrm{m}$ at $860 \mathrm{~A}$.

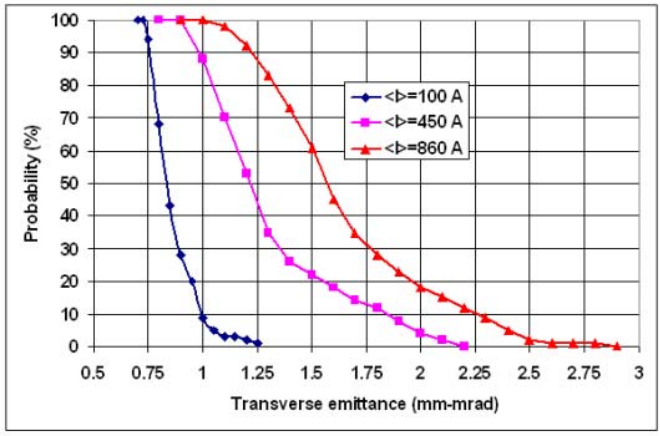

Figure 8: Probability vs emittance over 100 simulations.

The distribution of the values of the projected normalized brightness in 100 runs gives the results reported in table 3 showing that the compression gives an increase, as expected, not only of the average value but also of the standard deviation with a consequent reduction of the brightness stability.

Table 3: Projected normalized brightness in 100 runs

\begin{tabular}{|c|c|c|}
\hline $\begin{array}{c}\text { Current } \\
(\mathbf{A})\end{array}$ & $\begin{array}{c}<\mathbf{B n}> \\
\mathbf{A} /\left[(\mathbf{m}-\mathbf{r a d})^{2}\right]\end{array}$ & $\begin{array}{c}\boldsymbol{\sigma} \\
\mathbf{A} /\left[(\mathbf{m}-\mathbf{r a d})^{2}\right]\end{array}$ \\
\hline 100 & $1.1 \cdot 10^{13}$ & $0.25 \cdot 10^{13}$ \\
\hline 450 & $2.4 \cdot 10^{13}$ & $0.86 \cdot 10^{13}$ \\
\hline 860 & $2.8 \cdot 10^{13}$ & $1.2 \cdot 10^{13}$ \\
\hline
\end{tabular}

Concerning the slice emittance, in all simulations it does not exceed $1 \mu \mathrm{m}$ for the central slices at $450 \mathrm{~A}$ and 1.2 $\mu \mathrm{m}$ at $860 \mathrm{~A}$.

\section{CONCLUSIONS}

The results of the RF compressor optimization study in the SPARX injector show that a high peak current beam (up to $1 \mathrm{KA}$ in the slice) can be produced with a charge uniformly distributed through the pulse and a tolerable sensitivity on incoming phase jitter. A sensitivity study to various types of random errors in some significant operating points shows that the projected and slice emittance remain respectively below $2.5 \mu \mathrm{m}$ and $1.2 \mu \mathrm{m}$ in realistic operation conditions.

\section{REFERENCES}

[1] M. Boscolo et al. "Start To End Simulations for The SPARX Project", this Conference

[2] SPARC collaboration "Status of the SPARC Project", this Conference

[3] M. Ferrario et al. "Beam dynamics study of an RF bunch compressor for High Brightness beam injectors", Proceedings of EPAC02, p. 1762, June 2002 Paris [4] S.G. Anderson et al. "Velocity bunching of high brightness electron beams", Phys. Rev. ST Accel. Beams 8, 014401 (2005)

[5] J. Billen, "PARMELA", LA-UR-96-1835, 1996

[6] M. Biagini et al. "SPARC photoinjector working point optimization, tolerances and sensitivity to errors", Proceedings of EPAC04, p.396, July 2004 Lucerne 